\title{
Dynamics of concentrated colloidal suspensions probed by $x$-ray correlation spectroscopy
}

\author{
O. K. C. Tsui and S. G. J. Mochrie \\ Department of Physics, Massachusetts Institute of Technology, Cambridge, Massachusetts 02139-4307
}

(Received 28 July 1997)

\begin{abstract}
We report x-ray intensity fluctuation spectroscopy (XIFS) measurements of the equilibrium dynamics of colloidal suspensions of charge-stabilized $\mathrm{Sb}_{2} \mathrm{O}_{5}$ spheres of radius $250 \pm 40 \AA$ in glycerol. The dynamics were investigated for times between 4 and several thousand seconds, and for wave vectors, $Q$, from 0.003 to 0.012 $\AA^{-1}$, well beyond wave vectors that can be studied with visible light. The unique advantages of XIFS for studies of concentrated suspensions composed of refractive-index-mismatched components have not previously been explored. Here, we study a dilute sample (sample I) and a concentrated sample (sample II). For sample I, the diffusion coefficient is independent of $Q$, with a value consistent with the Stokes-Einstein relation. For sample II (effective hard-sphere volume fraction, $\phi=0.18$ ), the diffusion coefficient exhibits a minimum at the peak of the structure factor, indicating modifications to Brownian behavior due to interparticle interactions. [S1063-651X(98)10802-4]
\end{abstract}

PACS number(s): $82.70 . \mathrm{Dd}, 61.10 . \mathrm{Ht}, 42.25 . \mathrm{Kb}$

The recent commissioning of high-energy, thirdgeneration synchrotrons-the European Synchrotron Radiation Facility (ESRF) in Grenoble, France, in 1994 and the Advanced Photon Source (APS) at Argonne National Laboratory in 1996, together with the imminent operation of SPring-8 in Japan-present new opportunities for x-ray research. At the ESRF, APS, and SPring-8, the use of undulators, in conjunction with the small ring emittance and high lepton energy, produces $\mathrm{x}$-ray beams that are several orders of magnitude more brilliant than those available at secondgeneration sources. One of the most exciting opportunities offered by the unprecedented brilliance is to investigate the dynamics of condensed matter on molecular length scales using $x$-ray intensity fluctuation spectroscopy (XIFS). IFS using laser light - also known as photon correlation spectroscopy (PCS) or dynamic light scattering (DLS) - has long been employed to investigate the dynamics of condensed matter on micrometer length scales in transparent media, and the principles are well known [1]: a sample is illuminated by coherent radiation, resulting in a speckle pattern that varies in time as a result of temporal fluctuations within the sample. Time autocorrelations of the speckle yield the sample's characteristic times. The novelty of the present work lies, in part, in the use of synchrotron $\mathrm{x}$ rays, rather than laser light, to carry out PCS [2-4]. This allows us to examine smaller length scales than is possible with light scattering. Neutron spin-echo allows a similar range of wave vectors; however, it is restricted to time scales faster than $10^{-7} \mathrm{~s}$. XIFS on the other hand offers studies of slower dynamics with characteristic times of $10^{-4} \mathrm{~s}$ and longer [3].

XIFS has recently been demonstrated in pioneering studies of dilute colloidal suspensions of nanoscale gold particles protected from flocculation by fish gelatin [2], and of fractally aggregated palladium particles, also with a stabilizing surfactant [3]. An XIFS study of the diffusion of polymer micelles was reported in Ref. [4]. In the present paper, we examine the dynamics of smaller and simpler particles. Our main goal here is to show the unique capabilities of XIFS in studies of concentrated suspensions with significant van der Waals interactions between the particles of the suspension, and to elucidate any consequential effects on the dynamics. For particles with large van der Waals interactions, light scattering studies of the mode structure are not possible as a result of strong multiple scattering. This is because the existence of large van der Waals interactions accompanies a large refractive index mismatch between the colloidal particles and the suspending fluid [5].

Concentrated suspensions are of widespread practical interest. Examples include paints, inks, cosmetics, and certain foods [6]. Fundamental interest in the structure, phase behavior, and dynamics of colloids dates from the 1900s [7] and continues today $[6,5]$. In recent years, a good understanding has been achieved of the short-time diffusion of concentrated suspensions of hard spheres [8-10], particles with longranged Coulomb interactions [11], and particles with screened Coulomb interactions $[12,13]$. In contrast, the dynamics of suspensions having an attractive interaction have hardly been studied, including most especially systems realizing the DLVO interaction, which is composed of a van der Waals attraction and a screened electrostatic repulsion, and which is generic to colloidal systems from clays to proteins [6].

Our samples consisted of charge-stabilized $\mathrm{Sb}_{2} \mathrm{O}_{5}$ spheres of radius $250 \pm 40 \AA$ in glycerol at $\sim-30^{\circ} \mathrm{C}$. The dynamics were investigated for times between 4 and several thousand seconds, and for wave vectors $Q$ from 0.003 to 0.012 $\AA^{-1}$, well beyond what can be studied with visible light. To prepare the sample, we started with a $20 \%$-volume-fraction suspension in water purchased from Polysciences Inc., PA. It was twice concentrated by centrifugation and twice resuspended in glycerol by ultrasonication. Glycerol was chosen because it slows down the dynamics, thus permitting timeresolved measurements using an areal charge-coupled device (CCD) detector, realizing a large improvement in signal-tonoise ratio by averaging over a large number of CCD pixels $[2,14]$. A final centrifugation yielded the concentrated sample (sample II) with an effective hard sphere volume fraction of 0.18 . The dilute sample (sample I), with an effective hard sphere volume fraction of less than 0.02 , was drawn from the supernatant. The samples were spread across 
a $0.3 \mathrm{~mm}$ hole in a $0.2 \mathrm{~mm}$ thick stainless steel plate, which was supported in an evacuated sample holder and thermoelectrically cooled to $-31.5 \pm 0.3{ }^{\circ} \mathrm{C}$ and $-26.8 \pm 0.2{ }^{\circ} \mathrm{C}$ for samples I and II, respectively.

A partially coherent $\mathrm{x}$-ray beam was prepared and characterized at wiggler beamline X25 at the National Synchrotron Light Source (NSLS) [15]. In brief, $7 \mathrm{keV} x$ rays within a full width at half maximum (FWHM) bandwidth of $\Delta \lambda / \lambda=0.015$ were selected by a tungsten-boron carbide multilayer pair, located $18 \mathrm{~m}$ from the 27-pole wiggler source. Adjustable slits at $25 \mathrm{~m}$ were set to $d_{h}=4.2 \mu \mathrm{m}$ in the horizontal and $d_{v}=8.9 \mu \mathrm{m}$ in the vertical, so that the transverse beam dimensions were smaller than the transverse coherence lengths calculated for the NSLS source dimensions. Passing through the slits were $\sim 4 \times 10^{7}$ photons per second. As explained in Ref. [16], under the reasonable assumption that the energy spectrum may be approximated as a Lorentzian, the longitudinal coherence length is $\lambda^{2} / \pi \Delta \lambda$. The criterion requiring that the maximum optical path length difference in the sample be less than the longitudinal coherence length $\left(\lambda^{2} / \pi \Delta \lambda\right)$, leads to the condition $\lambda^{2} / \pi \Delta \lambda$ $\gtrsim 2 W \sin ^{2} \theta+d \sin 2 \theta$, where $W$ is the sample thickness, $d$ is the vertical or horizontal slit dimension for scattering in the vertical or horizontal direction respectively, and $2 \theta$ is the scattering angle, which was satisfied for wave vectors only up to $\sim 0.0015 \AA^{-1}$, which is 8 times smaller than the maximum wave vector studied in this work $\left(0.012 \AA^{-1}\right)$. The success of the present experiment shows that the aforementioned coherence criterion is too stringent to access the feasibility of XIFS experiments. In fact, this criterion would lead to a nearly fully coherent beam with a speckle contrast (b) close to unity. In the present experiment, however, we will show that sensible results are obtained for $b \simeq 0.2$ (see Fig. 2 below).

The sample was $80 \mathrm{~mm}$ downstream of the slits, contained within an evacuated sample holder with kapton windows for $\mathrm{x}$-ray access. Scattered $\mathrm{x}$ rays were collected using a Princeton Instruments CCD camera, employing direct $\mathrm{x}$ ray detection, located a distance $2.5 \mathrm{~m}$ downstream of the sample. The CCD pixel size was $22 \times 22 \mu \mathrm{m}^{2}$, which is less than the coherence area of the scattering, i.e., less than the speckle size of $\sim 45 \mu \mathrm{m}$ (horizontal) by $\sim 90 \mu \mathrm{m}$ (vertical) - ensuring that the detection scheme preserved the instantaneous intensity fluctuations [15]. Two-dimensional, time-resolved scattering data were acquired as a series of 900 6.00 and $4.00 \mathrm{~s} \mathrm{CCD} \mathrm{exposures} \mathrm{for} \mathrm{samples} \mathrm{I} \mathrm{and} \mathrm{II,} \mathrm{respec-}$ tively, each separated by $0.61 \mathrm{~s}$ for readout and storage. Individual exposures comprised $403 \times 653$ pixels.

The scattering cross sections for samples I and II, obtained by circularly averaging CCD images within rings 15 pixels wide and subtracting fitted backgrounds, are shown in Fig. 1(a) as solid and open circles, respectively. For sample I, the cross section decreases monotonically with increasing wave vector. By contrast, the scattering from sample II exhibits a peak at $0.008 \AA^{-1}$, corresponding to pronounced interparticle correlations-that is, the colloidal particles show liquidlike order. The scattering cross section may be described by

$$
V^{-1} d \sigma / d \Omega=r_{0}^{2} n\left(\rho_{p}-\rho_{m}\right)^{2} v^{2}|F(Q)|^{2} S(Q),
$$

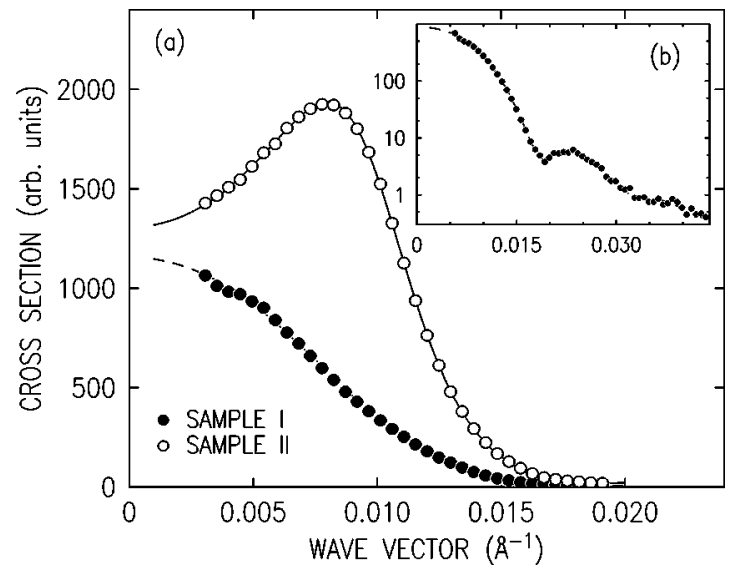

FIG. 1. (a) Scattering cross section vs wave vector for sample I at $-31.5^{\circ} \mathrm{C}$ (solid circles) and sample II at $-26.8^{\circ} \mathrm{C}$ (open circles). The solid lines show the model discussed in the text. Each data set is scaled arbitrarily for clarity of display. (b) Scattering cross section vs wave vector for sample I shown on a logarithmic intensity scale. The solid line is the model discussed in the text.

where $r_{0}$ is the Thomson radius, $n$ is the number density of colloidal particles, $\rho_{p}$ and $\rho_{m}$ are the electronic densities of $\mathrm{Sb}_{2} \mathrm{O}_{5}$ and glycerol, respectively, $F(Q)$ is the average form factor, $v$ is the volume of the spheres, and $S(Q)$ is a fluid structure factor, containing the effects of interparticle correlations.

For sample I, no interparticle correlations are apparent and the observed scattering may be fit with $S(Q)=1$. We thus infer that the $\mathrm{Sb}_{2} \mathrm{O}_{5}$ volume fraction is less than 0.02 . The best fit to data extending to $0.04 \AA^{-1}$ [Fig. 1(b)] indicates a mean sphere radius of $R=250 \AA$ and a standard deviation $\sigma=40 \AA$ A. For sample II, we fit the data to the form of $S(Q)$ for hard spheres within the Percus-Yevick approximation [17]. Fits were performed with $R$ held at $250 \AA$ for $F(Q)$, yielding an effective hard-sphere radius, $R_{S}=281 \AA$, and an effective volume fraction, $\phi=0.18$. The fact that the fitted value of $R_{S}$ is only $\sim 12 \%$ larger than the size of the spheres $R$ may suggest the existence of an effectively screened Coulomb repulsion between the particles [12]. Because $S(Q)$ is not very sensitive to the interparticle potential and because there is some uncertainty in the fitted background, the high quality of the fit in Fig. 1(a) should not be taken to imply that the $\mathrm{Sb}_{2} \mathrm{O}_{5}$ spheres interact via a hardsphere potential. In fact, one expects their interaction to be of the DLVO type [18].

To determine the variation of the diffusion time versus wave vector, we first calculated the normalized autocorrelation, $g_{2}(t)$, for each CCD pixel as a function of the delay time $t$ :

$$
g_{2}(t)=(n-t)^{-1} \sum_{s=1}^{n-t} i_{s} i_{s+t} / \bar{i}_{s} \bar{i}_{s+t}
$$

where $n$ is the number of time steps, $i_{s}$ and $i_{s+t}$ are the intensities at time steps $s$ and $s+t$, respectively, and $\bar{i}_{s}$ and $\bar{i}_{s+t}$ are the average intensities at the wave vector in question at time steps $s$ and $s+t$, respectively $[2,14]$. Second, we averaged $g_{2}(t)$ over all pixels within rings of width $0.00031 \AA^{-1}$ (10 pixels), $0.00047 \AA^{-1}$ (15 pixels), or 


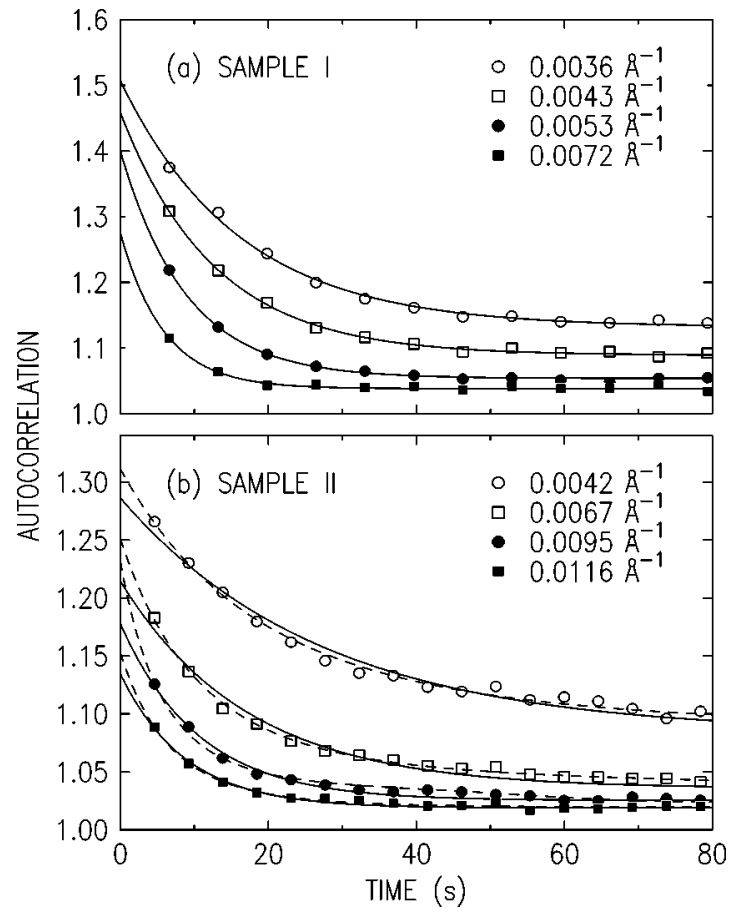

FIG. 2. (a) Autocorrelation vs time for sample $\mathrm{I}$ at $-31.5^{\circ} \mathrm{C}$ at four wave vectors. Solid lines correspond to an exponential decay vs time. (b) Autocorrelation vs time for sample II at $-26.8{ }^{\circ} \mathrm{C}$ at four wave vectors. Solid lines correspond to an exponential decay vs time. The dashed lines correspond to fits to the cumulant expression Eq. (4).

$0.00093 \AA^{-1}$ (30 pixels), for wave vectors smaller than $0.005 \AA^{-1}$, between 0.005 and $0.010 \AA^{-1}$, or larger than $0.010 \AA^{-1}$, respectively. The resultant autocorrelations for sample I are shown versus time at four wave vectors in Fig. 2(a). It is apparent that these data are of high quality, enabling unambiguous determination of the characteristic times of the sample.

To quantify the experimental autocorrelations of Fig. 2, we have fit the data at each wave vector to a single exponential decay:

$$
g_{2}(t)=a+b e^{-2 t / \tau} Q
$$

This model, shown as the solid lines in Fig. 2(a), provides a good description of the data. The fitted values of the baseline, $a$, are close to unity, except at small wave vectors, where the signal of interest suffers a non-circularlysymmetric background produced by scattering from the slits. The zero-time intercept $b$ is consistent with an analysis of the visibility of static speckle patterns [15], indicating the absence of thermal motions occurring on shorter time scales.

Shown in Fig. 2(b) are autocorrelations versus time for sample II at four representative wave vectors. The solid lines in this figure correspond to fits to the same model [Eq. (3)], which, however, provide a poorer description of the autocorrelations than for sample I, especially at smaller wave vectors. It is then better to characterize the decay in terms of the cumulants $[1]$ :

$$
g_{2}(t)=a+b e^{-2 t / \tau_{Q}+c_{2}\left(2 t / \tau_{Q}\right)^{2} / 2-c_{3}\left(2 t / \tau_{Q}\right)^{3} / 6},
$$

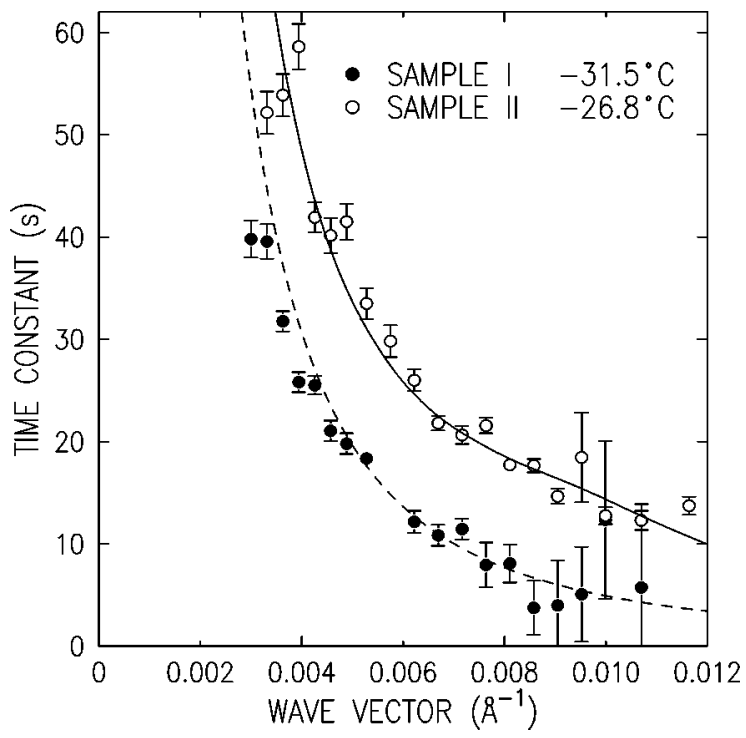

FIG. 3. Time constants for sample I at $-31.5^{\circ} \mathrm{C}$ (solid circles) and sample II at $-26.8^{\circ} \mathrm{C}$ (open circles). The lines are guides to the eye.

where $2 / \tau_{Q}, c_{2}$, and $c_{3}$ may be interpreted as the mean, the relative variance, and the relative skewness, respectively, of the decay rate distribution [1]. It is noteworthy that the value of $2 / \tau_{Q}$ so determined also equals the initial decay rate and is related to the short-time diffusion coefficient $D$ via $D$ $=1 /\left(\tau_{Q} Q^{2}\right)$. The resultant best fits, which provide a good description of the data, are shown in Fig. 2(b) as dashed lines. A distribution of particle sizes could give rise to a distribution of decay rates. However, the fact that the autocorrelations of sample I are well described by single exponentials indicates instead that interparticle interactions originate this behavior. To this end, it has been proposed that the initial exponential decay corresponds to diffusion of a particle within a region bounded by the neighboring particles, and that the slower variation at longer times involves escape from this region [9]. This interpretation seems consistent with the observation that the non-single-exponential behavior is more pronounced at smaller wave vectors, where the characteristic time of the dynamics is longer.

In Fig. 3, the fitted time constants for sample I using the single-exponential model and for sample II using the cumulant form are shown as solid and open circles, respectively. In both cases, the time constant is largest at the smallest wave vectors and decreases with increasing wave vector. There is, however, an important difference between the behavior of the two samples, highlighted in Fig. 4(a), which plots $D_{0} / D$ versus wave vector, where $D_{0}$ $=k_{B} T / 6 \pi \eta(T) R$ is the diffusion coefficient of an isolated sphere of radius $R$ in pure glycerol [6]. [ $\eta(T)$ is the viscosity of pure glycerol $[19] \eta\left(-31.5 \pm 0.3^{\circ} \mathrm{C}\right)=7310 \pm 420 \mathrm{P}$ and $\eta\left(-26.8 \pm 0.2^{\circ} \mathrm{C}\right)=3290 \pm 140 \mathrm{P}$.] For sample I, $D_{0} / D$ is nearly constant versus wave vector, as expected for a dilute suspension of spheres undergoing Brownian diffusion. We estimate that a water content of about $3 \%$ would decrease the liquid viscosity from that of pure glycerol by a factor of about 2.5, changing $D_{0} / D$ from 1 to the observed value of 0.38 . In contrast to the behavior for sample I, the value of $D_{0} / D$ for sample II shows a significant wave-vector depen- 


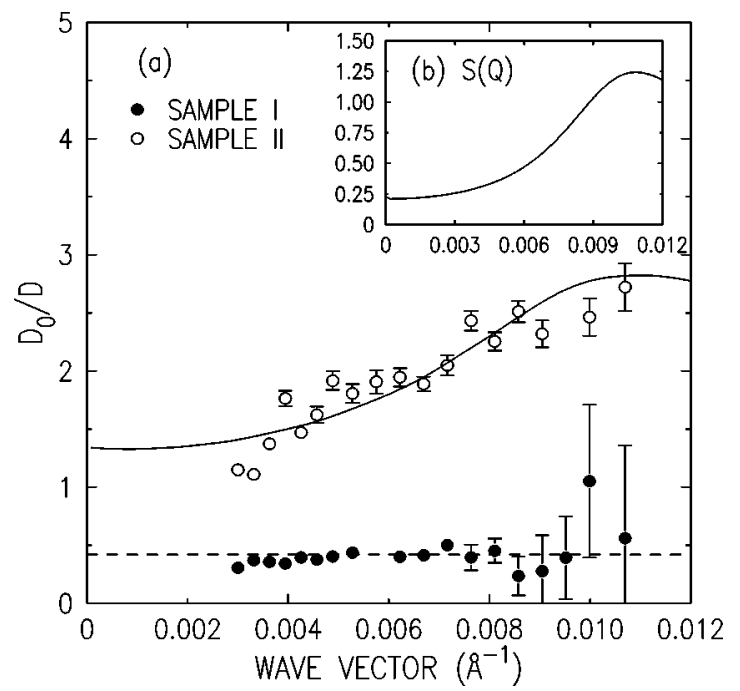

FIG. 4. $D_{0} / D$ vs wave vector for sample I at $-31.5^{\circ} \mathrm{C}$ (solid circles) and sample II at $-26.8^{\circ} \mathrm{C}$ (open circles). The dashed line corresponds to $D_{0} / D=0.38$. The solid line is a guide to the eye.

dence, increasing from $\sim 1.2$ at $0.003 \AA^{-1}$ to $\sim 2.5$ near $0.011 \AA^{-1}$, where the structure factor peaks [Fig. 4(b)]. Slower diffusion for wave vectors approaching the peak of the structure factor $\left(Q_{m}\right)$ has been observed previously in light scattering studies of suspensions of interacting colloidal particles $[20,11,8,9]$.

The effective diffusion coefficient of a concentrated colloidal suspension, derived from the initial decay of the autocorrelation, is generally written as $D(Q)=D_{0} H(Q) / S(Q)$ $[9,10,12,13]$, where the function $H(Q)$ encompasses the effect of hydrodynamic interactions-derived from the velocity field in the suspending fluid created by the neighboring particles-and $S(Q)$ is the static structure factor that accounts for the effect of direct interparticle interactions. For dilute suspensions, $H(Q)$ and $S(Q)$ equal unity [11]. For concentrated suspensions with hard sphere $[9,10]$ or screened Coulomb $[12,13]$ interactions, however, $H(Q)$ shows a peak at $Q_{m}$ with a value that is less than one for hard spheres and approximately one for charged particles. By contrast, the peak in $S(Q)$ at $Q_{m}$ is more pronounced. As a result, the structure factor always dominates the dynamics around $Q_{m}$ in these systems, giving rise to a minimum in $D$ at $Q_{m}$. In Fig. 4(b) is shown the structure factor for sample II inferred from the fits to the data of Fig. 1. Evidently, $S(Q)$ increases by a factor of about 5 as the wave vector increases from zero to $Q_{m}$. In the same wave vector range, $D_{0} / D$ rises only by a factor of 2 [Fig. 4(a)]. We thus infer that $H(Q)$ for sample II shows a weaker peak at $Q_{m}$ than $S(Q)$, similar to what was observed in hard-sphere and charged-particle suspensions, as noted above. Physically, for the density wave characterized by wave vector $Q_{m}$, the particles are separated by the optimum distance, so that any attempt to move the particles away from this preferred configuration is suppressed.

The other notable difference between sample I and sample II is that the collective diffusion coefficient, given by the small- $Q$ limit of $D$, is smaller by a factor of about 3 for sample II than for sample I. In contrast, light scattering studies of hard-sphere-like particles find that collective diffusion increases weakly with increasing concentration $[8,9]$. In the case of charged suspensions with repulsive Coulomb interactions, the collective diffusion coefficient also increases with increasing concentration. It is natural to suppose that the different behavior we observe is the result of attractive van der Waals interactions among the $\mathrm{Sb}_{2} \mathrm{O}_{5}$ spheres [21]. Further studies will elucidate this point.

In conclusion, we have demonstrated that XIFS is able to study dynamical behavior of concentrated colloidal suspensions with large van der Waals interactions in which diffusion parameters are considerably modified by interparticle interactions. The prospects for XIFS are exciting indeed. Besides its capability to access molecular length scales, the opportunity offered by XIFS for the investigation of the dynamics of concentrated suspensions with refractive-indexmismatched constituents is unprecedented. For example, it will be possible to extend to van der Waals systems the beautiful measurements on the behavior near the glass transition carried out for hard-sphere colloids by van Megen and Underwood [22]. In view of the emerging realization that the range of interparticle potentials is extremely important in determining the phase behavior-specifically, sufficiently long-ranged attractive potentials are necessary for existence of a stable liquid-gas critical point [5] — such studies will be particularly interesting.

We are very grateful to D. Abernathy, L. Berman, S. Brauer, S. Dierker, E. Geissler, G. Grübel, A. Lomakin, L. Lurio, A. Mayes, I. McNulty, L. Regan, A. Sandy, B. Stephenson, M. Sutton, Z. Yin, and M. Yoon for invaluable discussions, advice, and assistance. This work was supported by the NSF (Grant No. DMR-9312543). The NSLS and X25 were supported by the DOE (Grant No. DEAC0276CH00016).
[1] B. Chu, Laser Light Scattering (Academic Press, Orlando, FL, 1974).

[2] S. Dierker, R. Pindak, R. M. Fleming, I. K. Robinson, and L. E. Berman, Phys. Rev. Lett. 75, 449 (1995).

[3] T. Thurn-Albrecht, W. Steffen, A. Patkowski, G. Meier, E. W. Fischer, G. Grübel, and D. L. Abernathy, Phys. Rev. Lett. 77, 5437 (1996).

[4] S. G. J. Mochrie, A. M. Mayes, A. R. Sandy, M. Sutton, S. Brauer, G. B. Stephenson, D. L. Abernathy, and G. Grübel,
Phys. Rev. Lett. 78, 1275 (1997).

[5] W. C. K. Poon and P. N. Pusey, in Observation, Prediction, and Simulation of Phase Transitions in Complex Fluids, edited by M. Baus et al. (Kluwer, Dordrecht, 1995).

[6] R. J. Hunter, Introduction to Modern Colloid Science (Oxford University Press, Oxford, 1993).

[7] A. Einstein, Investigations on the Theory of Brownian Movement (Dover, New York, 1956).

[8] W. van Megen, R. H. Ottewill, S. M. Owens, and P. N. Pusey, 
J. Chem. Phys. 82, 508 (1985).

[9] P. N. Segrè, O. P. Behrend, and P. N. Pusey, Phys. Rev. E 52, 5070 (1995), and references therein.

[10] C. W. J. Beenakker and P. Mazur, Physica A 126, 349 (1984).

[11] J. C. Brown, P. N. Pusey, J. W. Goodwin, and R. H. Ottewill, J. Phys. A 8, 664 (1975).

[12] G. Nägele, M. Watzlawek, and R. Klein, Prog. Colloid Polym. Sci. 104, 31 (1997), and references therein.

[13] A. P. Philipse and A. Vrij, J. Chem. Phys. 88, 6459 (1988).

[14] A. P. Y. Wong and P. Wilzius, Rev. Sci. Instrum. 64, 2547 (1993).

[15] O. K. C. Tsui, S. G. J. Mochrie, and L. E. Berman, Synchroton Radiat. (to be published).

[16] Miles V. Klein, Optics (John Wiley and Sons, New York, 1970).

[17] J. K. Percus and G. J. Yevick, Phys. Rev. 110, 1 (1958).
[18] The refractive index of glycerol is 1.474 (Aldrich Catalog Handbook) and that of $\mathrm{Sb}_{2} \mathrm{O}_{5}$ should be about 3: we have been unable to find the refractive index of the material but we expect it to be similar to that of $\mathrm{Sb}_{2} \mathrm{O}_{4}$, which is 3.1 [L. Gmelin, Handbuch der Anorganischen Chemie 8th ed. (Verlag Chemie, Berlin, 1924)]. According to Ref. [6], our sample is likely to realize DLVO interactions.

[19] G. Tammann and W. Hesse, Z. Anorg. Allg. Chem. 156, 17 (1926).

[20] D. W. Schaefer and B. J. Berne, Phys. Rev. Lett. 32, 1110 (1974).

[21] A. R. Altenberger, J. Chem. Phys. 70, 1994 (1979); A. R. Altenberger and J. M. Deutch, ibid. 59, 894 (1973).

[22] W. van Megen and S. M. Underwood, Phys. Rev. Lett. 70, 2766 (1993); W. van Megen and S. M. Underwood, Phys. Rev. E 49, 4206 (1994). 